\title{
CITATIONS INDEX
}

\begin{tabular}{|c|c|c|c|c|c|}
\hline & 1. Bible & $24: 24$ & 333 & $32: 23$ & $36,41,42$, \\
\hline & Gen. & $24: 29$ & 333 & & 315,318 \\
\hline $9: 21$ & 49 & $24: 35$ & $36,42,157$ & $33: 1$ & $42,316,318$ \\
\hline $12: 16$ & $36,41,42,157$ & $25: 1$ & 90, 99 & $33: 2$ & 42,315 \\
\hline $14: 14$ & $63,64,68,78,79$ & $25: 6$ & 92,9399 & $33: 6$ & 42,316 \\
\hline $15: 2$ & 79,80 & $25: 9$ & 54 & $34: 14$ & 164 \\
\hline $15: 3$ & 79,80 & $25: 12$ & 42,56 & $34: 25$ & 100 \\
\hline $16: 1$ & $36,42,170$ & $25: 19$ & 56 & Ch. 35 & 35 \\
\hline $16: 2$ & 42 & $25: 32$ & 101 & $35: 22$ & $89,90,95$ \\
\hline $16: 2 \mathrm{ff}$ & 314 & $28: 8$ & 135 & & $97,99,104,105$, \\
\hline $16: 3$ & 36,42 & $28: 22$ & 331 & & $107-109,119,315$ \\
\hline $16: 5$ & 42 & Ch. 29 & 335 & $35: 25-6$ & $42,315,316$ \\
\hline $16: 6$ & 42 & $29: 5$ & 333 & $36: 12$ & $89,108,323$ \\
\hline $16: 8$ & 42 & $29: 24$ & $42,314,336,337$ & Ch. 37 & 316 \\
\hline Ch. 17 & $65,68,76,93$ & $29: 29$ & $36,40,42,314$ & $37: 2$ & 56,317 \\
\hline $17: 12$ & $62,68,78$ & $29: 32-30: 24$ & $4 \quad 339$ & $39: 10$ & 109 \\
\hline $17: 20$ & 55 & Ch. 30 & 315,342 & $42: 22$ & 176 \\
\hline $17: 21$ & 55 & $30: 3$ & $36,41,42$, & $42: 27$ & 67 \\
\hline $17: 23$ & 62 & & 315,317 & $43: 9$ & 163 \\
\hline $17: 27$ & 62,68 & $30: 3-13$ & 314 & Ch. 46 & 335 \\
\hline Ch. 19 & 113 & $30: 4$ & $36,42,157$ & $46: 18$ & 315,316 \\
\hline $19: 8$ & 113 & $30: 5$ & 56 & $46: 25$ & 315,316 \\
\hline $19: 30-38$ & 100 & $30: 7$ & 42 & $48: 5$ & 332 \\
\hline $20: 14$ & $36,42,157$ & $30: 9$ & $36,42,317$ & Ch. 49 & 106,109 \\
\hline $20: 17$ & 42,324 & $30: 10$ & 42,342 & $49: 3-4$ & 99 \\
\hline $21: 2$ & 56 & $30: 12$ & 42,342 & $49: 4$ & $106,108,109,111$ \\
\hline 21:9-10 & 54 & $30: 18$ & 42 & $49: 5-7$ & 100 \\
\hline $21: 10$ & $36,42,54$ & $30: 23$ & 164,217 & & \\
\hline $21: 12$ & 36,42 & $30: 43$ & $36,42,167$ & & Exod. \\
\hline $21: 13$ & $36,42,54$ & $31: 15$ & 67,68 & $1: 13$ & 126 \\
\hline $22: 3$ & 113 & $31: 33$ & $36,42,318$ & $2: 4-11$ & 127 \\
\hline $22: 10$ & 113 & $31: 50$ & 343 & $2: 5$ & 42 \\
\hline $22: 24$ & 89,115 & $32: 5$ & 42 & $11: 5$ & 42 \\
\hline $24: 15$ & 333 & $32: 6$ & 36,42 & $12: 29$ & 113 \\
\hline
\end{tabular}




\begin{tabular}{|c|c|c|c|c|c|}
\hline $12: 36$ & 232,320 & $21: 20-21$ & 12,67 & & $168,169,172-175$, \\
\hline $12: 43-45$ & 67 & $21: 21$ & 66,67 & & $177,181,183$ \\
\hline $12: 43$ & 68 & $21: 23-27$ & 12 & & $216,217,227$, \\
\hline $12: 44$ & 68 & $21: 25-26$ & 222 & & $251,255,262-265$, \\
\hline $12: 45$ & 238 & $21: 26-27$ & $4,36,123$ & & $267,268,273-276$, \\
\hline $20: 10$ & $36,42,60,61$ & & $152,226,350$ & & $285,288,296$ \\
\hline $20: 14$ & 36,42 & $21: 32$ & 36,42 & & $299,346,349$ \\
\hline \multirow[t]{3}{*}{ Ch. 21} & $12,23-25$ & $22: 2$ & 221 & $19: 20-21$ & 10, 13, 14, \\
\hline & $124,128,140$ & $22: 4$ & 192,193 & & $33,34,349$ \\
\hline & 155,227 & Ch. 23 & 61 & $19: 20-22$ & $37,151,156$ \\
\hline $21: 2$ & $28,129,221$ & $23: 11$ & 192 & & $158,175,217$ \\
\hline $21: 2-6$ & $20,129,222,226$ & $23: 12$ & $42,60,61,62$ & & 251,260 \\
\hline \multirow[t]{4}{*}{$21: 2-11$} & $33,53,121$ & $23: 24$ & 193 & $19: 21$ & 283 \\
\hline & $122,127,130$, & $31: 17$ & 60 & $19: 22$ & 285 \\
\hline & $132-134,137$ & $32: 1-20$ & 109 & $19: 23$ & 157 \\
\hline & $141,150,347$ & $32: 2$ & 193 & $20: 12$ & 270 \\
\hline $21: 2-27$ & 134 & $32: 11$ & 84 & $20: 13$ & 265 \\
\hline $21: 3$ & 132 & & & $20: 17$ & 270 \\
\hline \multirow[t]{4}{*}{$21: 4$} & $9,33,127$ & & Lev. & $22: 11$ & $65,75,76$ \\
\hline & $132,150,152$ & $5: 6$ & 156 & $23: 24$ & 329 \\
\hline & $218,221,297$ & $5: 14-16$ & 175 & Ch. 25 & $28,221,222$ \\
\hline & 304 & $5: 15$ & 156,283 & $25: 6$ & 36,42 \\
\hline $21: 4-5$ & 37 & $5: 17-19$ & 175 & $25: 10$ & 221 \\
\hline $21: 4-6$ & 141 & $5: 20-25$ & 175 & $25: 39$ & $13,221,229$ \\
\hline 21:4-11 & 153,333 & $5: 20-26$ & 175 & $25: 39-46$ & $21,125,347$ \\
\hline $21: 5-6$ & 309 & $5: 22$ & 175 & $25: 42$ & 66,226 \\
\hline $21: 6$ & $128,129,309$ & $5: 50$ & 166 & $25: 43$ & 13 \\
\hline $21: 7$ & $42,43,169$ & $13: 36$ & $\begin{array}{l}100 \\
176\end{array}$ & $25: 44$ & $36,129,221$, \\
\hline \multirow{8}{*}{ 21:7-11 } & $219,221,226-228$ & $15: 18$ & 109,263 & & 226,227 \\
\hline & $21,22,23$ & $15: 24$ & 262,264 & $25: 44-45$ & $222,237,240$ \\
\hline & $29,37,128$ & $17: 1-26: 46$ & 128 & $25: 44-46$ & 220 \\
\hline & $132,138,139$ & Ch. 18 & 103 & $25: 45$ & 238,239 \\
\hline & $141,142,150$ & $18: 3$ & 103 & $25: 46$ & 243 \\
\hline & $160,224,225$ & $18: 9$ & 68 & $25: 47-50$ & 128,341 \\
\hline & $228,346,349$ & $18: 11$ & $\begin{array}{r}68 \\
270\end{array}$ & $25: 47-55$ & 127 \\
\hline & $37,66,68$ & $18 \cdot 15$ & $\begin{array}{r}270 \\
131 \quad 270\end{array}$ & $25: 51$ & 274 \\
\hline \multirow{4}{*}{$21: 8$} & (20, & 10.10 & 131, 270 & $25: 55$ & 3 \\
\hline & $129,139,143$, & $18: 20$ & 156 & $26: 30$ & 193 \\
\hline & 144, 148, 160, & $18: 26$ & $\begin{array}{r}269 \\
\end{array}$ & $27: 33$ & 176 \\
\hline & 222 & Ch. 19 & $40,153,156,157$ & & \\
\hline $21: 9$ & 128 & $19: 20$ & $13,14,22-24$ & & \\
\hline $21: 10$ & 139,150 & & $29,40,41,42$ & & Num. \\
\hline 21:11 & 228 & & $43,94,109$ & $1: 18$ & 78 \\
\hline $21: 12$ & 12 & & $133,152,154$ & $5: 6-7$ & 158,159 \\
\hline $21: 19$ & 67 & & $155,158-161$ & $5: 6-8$ & 175 \\
\hline $21: 20$ & $13,36,350$ & & $162,163,166$ & $5: 13$ & 109,156 \\
\hline
\end{tabular}




$\begin{array}{lrl}6: 24-26 & 110 & 23: 18 \\ \text { Ch. } 7 & 329 & 24: 1 \\ 7: 3 & 330 & 25: 19 \\ 12: 5 & 85 & 28: 30 \\ 12: 7 & 86 & 28: 68 \\ 12: 7-8 & 42 & 29: 7 \\ 13: 22 & 68 & 33: 6 \\ 13: 28 & 68 & 34: 5 \\ 21: 20 & 87 & \end{array}$

5:12-18

5:14

$5: 18$

$7: 1-3$

7:3

$7: 4$

$12: 12$

12:18

Ch. 15

15:1-3

15:12

15:12-18

15:14

15:17

16:11

16:14

20:16

20:16-17

21:10-14

21:14

Ch. 22

22:9-11

22:14ff

22:22

22:23

22:25-27

$23: 4$

23:8-9
Deut.

$\begin{array}{rl} & 5: 9 \\ 163 & 9: 21 \\ 36,46 & 15: 14 \\ 36,46 & \\ 220,238 & \\ 78 & 1: 1-2 \\ 78 & 5: 18 \\ 36,42 & 8: 31 \\ 36,42 & 9: 2\end{array}$

128, 129, 132, Ch. 9 140, 142, 229

24

129, 219, 221,

226, 227, 229,

230, 267

21, 121, 124, 127, 136, 347

268

$36,37,42$, 128, 226, 227,

309

$$
36,42
$$

42

39, 243

220, 238

221

66, 136

$156 \quad 19: 9$

$156 \quad 19: 19$

$156 \quad 19: 27$

$151,153,160 \quad 19: 27-28$

133, 152, $160 \quad 20: 4$

$161 \quad 20: 18$

$239 \quad 21: 25$

$239 \quad 22: 23$
269, 324

272-276 1:11

$108 \quad 1: 16$

$320 \quad 1: 18$

$42 \quad 8: 16$

239, $243 \quad 10: 26$

$107 \quad 11: 7$

$42 \quad 14: 48$

$15: 3$

Josh.

$$
\text { Judges }
$$

$164 \quad 17: 10$

$239 \quad 17: 26$

$68 \quad 17: 36$

$17: 45$

$20: 6$

$117 \quad 20: 29$

$168 \quad 24: 27$

57, $94 \quad 24: 41$

$58 \quad 25: 24$

$58,121 \quad 25: 25$

$58 \quad 25: 28$

57, 90, $94249 \quad 25: 31$

$37 \quad 25: 39$

$117 \quad 25: 41$

$114 \quad 27: 12$

$94,111, \quad 28: 21$

113-116 28:22

98

90, 99, 100, 101, 111, 114, 117

90, 99, 115, 116

111

$90,115,116$

90, 96, 116

116

116

$116 \quad 14: 15$

37, $116 \quad 14: 16$

$5: 13$

$6: 20$

6:22

Ch. 13

13:1

$13: 13$

14:7

$14: 12$

$14: 16$
$14: 17$

$115,116,117 \quad 14: 17$

$112 \quad 14: 19$

$115 \quad 16: 21$

$117 \quad 16: 21-22$

$115 \quad 17: 19$

$152 \quad 20: 17$
1Sam.

37

37

37

37

115

113

109

109

109

164

164

164

164

47

47

37

37

37

37

37

37

174

37

309

37

37

2Sam.

90

37

37

100

110

164

37

37

37

37

37

37

100

90, 99

169

37 


\begin{tabular}{|c|c|c|c|c|c|}
\hline 21:11 & 89 & $34: 9$ & $36,37,45$ & $45: 10$ & 320 \\
\hline $21: 16$ & 68 & $34: 10,11,16$ & 36,37 & $55: 13$ & 163 \\
\hline \multirow[t]{3}{*}{$21: 18$} & 68 & $35: 5$ & 80 & $68: 5$ & 330 \\
\hline & & & & $68: 22$ & 158 \\
\hline & & \multicolumn{2}{|c|}{ Ezek. } & Ch. 86 & 59 \\
\hline $1: 13$ & 37 & Ch. 16 & 13 & $86: 3$ & 59 \\
\hline $1: 17$ & 37 & $22: 10$ & 100 & $86: 11$ & 59 \\
\hline $2: 22$ & 99 & Ch. 23 & 13,101 & $86: 16$ & 59,60 \\
\hline $3: 20$ & 37 & $23: 20$ & $90,91,100$ & $96: 13$ & 330 \\
\hline $3: 26$ & 68 & & 101,102 & $98: 9$ & 330 \\
\hline $3: 27$ & 68 & $23: 30$ & 98 & $116: 10$ & 59 \\
\hline \multirow[t]{3}{*}{$11: 3$} & 90 & $34: 11$ & 176 & $116: 16$ & 59 \\
\hline & & $34: 11-12$ & 176 & $120: 34$ & 333 \\
\hline & & & & $123: 2$ & 36,37 \\
\hline
\end{tabular}

$4: 2$

4:16

$5: 2$

$5: 4$

5:26

$14: 9$

$16: 15$

19:22

$4: 1$

$8: 3$

$13: 16$

$14: 2$

24:2

$37: 23$

43:9

$45: 18$

47:3

$54: 4$

\begin{tabular}{|c|c|c|}
\hline \multicolumn{3}{|c|}{ Jer. } \\
\hline $2: 4$ & 64 & $14: 2$ \\
\hline $2: 14$ & 62 & \\
\hline $3: 2$ & 320 & \\
\hline $5: 11$ & 145 & $2: 11$ \\
\hline $6: 10$ & 164 & \\
\hline $16: 5$ & 48 & \\
\hline $23: 32$ & 193 & Ch. 16 \\
\hline Ch. 34 & 273 & $27: 4$ \\
\hline
\end{tabular}

$6: 7$

Isa.
Hos.

\begin{tabular}{rrlrr} 
& 150 & \multicolumn{3}{c}{ Prov. } \\
& 164 & $14: 31$ & 163 \\
& & $20: 25$ & 176 \\
Joel & & $27: 22$ & 169 \\
& 36,37 & $30: 21-23$ & 4 \\
& & $30: 23$ & $37,43,227$
\end{tabular}

Amos

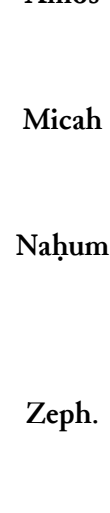

48

Job

$1: 3$

157

$14: 1$

$331 \quad \begin{array}{ll}15: 14 \\ 19: 5\end{array}$

68

68

37,163

$25: 4$

68

165, 170

$\begin{array}{ll}97 & 27: 6 \\ 37 & 29: 4\end{array}$

180

36,37

Cant.

$6: 8$

90

Ruth

ZeCh.

$\begin{array}{ll} & 2: 13 \\ 320 & 2: 14 \\ & 3: 9 \\ & 4: 11 \\ 145 & 4: 22\end{array}$

37

106

37

Mal.

4:11

315

115

Ps.

$59 \quad 3: 30$

164

341 
Eccl.

2. Bible Translations $21: 10$

122

$2: 7$

36,37

23:12

60

$8: 1$

9: 7-8

86

Septuagint (LXX)

150

Gen.

Lev.

$15: 2$

$15: 3$

19:20

151, 153, 161

Esth.

$21: 10$

79

25: 44

125

$7: 4$

36,37

21:13

Dan.

$30: 23$

54

54

164

89, 105, 106,

$35: 22$

89

$5: 3$

$95 \quad 38: 10$

106

$95 \quad 49: 4$

106, 109

19:20

173

Ezra

Exod.

$\begin{array}{lrl}2: 58 & 221 & 21: 7 \\ 2: 65 & 36,37 & 21: 10 \\ 4: 4 & 238 & 21: 26-27 \\ 10: 2-3 & 9 & \\ 10: 14 & 238 & \end{array}$

43, 227

150

$23 \cdot 20$

Ezek.

Jewish Bible Translations

Lev.

Neh.

19:20

2:6

$3: 26$

$7: 67$

13:23-24

$\begin{array}{rr} & 19: 20 \\ 320 & 25: 44 \\ 221 & \\ 36, \quad 37 & \\ 9 & 15: 12\end{array}$

43, 152, 160,

172,227

Targum Onkelos

227

$15: 2$

Gen.

22:26

80
320

Deut.

$31: 33$

318

$15: 12$

227

318

318

1Chron.

$1: 31$

1:32

$2: 1-2$

2:46

2:48

$5: 1$

$5: 1-3$

$7: 13$

4:18

14:4

$20: 4$

2Chr.

$\begin{array}{rl}99 & 23: 20 \\ 89,90 & \\ 316 & 5: 2-3 \\ 90 & 5: 23 \\ 90 & \\ 105 & \\ 106 & 15: 2 \\ 316,340 & \end{array}$

Ezek.

33:1-2

318

$33: 6$

89,324

Dan.

$102 \quad 35: 22$

$36: 12$

323

95, 319

95, 319

Peshitta

Gen.

21:6

Exod.

316,340

86

Samaritan Pentateuch

Gen.

$68 \quad 15: 3$

$49: 4$

$9: 18$

28:8-10

28:10

$$
\begin{array}{rr}
347 & \\
21 & \\
36,37 & 21: 4 \\
& 21: 8 \\
& 21: 9
\end{array}
$$

$\begin{array}{rl}79 & 18: 9 \\ 106,109 & 19: 20 \\ & 25: 45\end{array}$

Lev.

$$
\text { 123, } 309
$$

320

$22: 26$

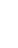


Targum Pseudo-Jonathan

\begin{tabular}{|c|c|c|}
\hline \multicolumn{2}{|c|}{ Gen. } & 19:20 \\
\hline $15: 2-3$ & 80 & $26: 30$ \\
\hline $20: 17$ & 324 & \\
\hline 21:12 & 56 & \\
\hline $29: 24$ & 343 & $15: 17$ \\
\hline $29: 29$ & 343 & $25: 5$ \\
\hline $30: 3$ & 317 & \\
\hline $30: 9$ & 317 & Sa \\
\hline 31:33 & 318 & \\
\hline $32: 23$ & 318 & $15: 3$ \\
\hline $33: 1-2$ & 318 & 21:10 \\
\hline 33:6 & 318 & \\
\hline $35: 22$ & 110 & \\
\hline $37: 2$ & 317 & 23:12 \\
\hline
\end{tabular}

Exod.

$12: 36$
$14: 21$
$21: 6$
$21: 10$
$22: 4$
$23: 11$
$23: 12$

19:20

$25: 45$

Lev.

$$
\text { 161, 172, } 275
$$$$
238
$$

Deut.

25:5

\section{Targum Neofiti}

Gen.

$22: 24$

$31: 33$

$32: 23$

$33: 1-2$

$33: 6$

$35: 22$

$37: 2$

Exod.

19:20

$9: 4$

$23: 32$

$15: 3$

$\begin{array}{ll}109 & 40: 17 \\ 318 & 71: 24 \\ 318 & \\ 318 & \\ 318 & 2: 7 \\ 324 & \\ 317 & \end{array}$

$27: 6$
Deut.

Lev. 162, 172, 275 192-193

$\begin{array}{ll}192-193 & 4 Q 215 \\ & 11 Q P 5 a\end{array}$

309

192

\section{Samaritan Targum}

Gen.

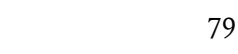

Exod.

60-61

\section{Dead Sea Scrolls}

4Q252 107

334

105
Lev.

\section{Apocrypha, Pseudo-Epigrapha \& Judeo-Hellenistic Works \\ Ahiqar}

58, 322, 323

Joseph and Aseneth

Ch. 24

56,316

Josephus Ant. $1: 78 \quad 65$

\section{Targum Jonathan}

162,172

1:303

317

193

$4: 244$

$5: 142$

90, 114

$5: 233$

249

2Sam.

323

Jer.

$\begin{array}{ll} & 13: 25 \\ 193 \quad 14: 2 \\ & 34: 15\end{array}$

Jubilees

\section{Fragment Targum}

Gen.

79

Letter of Aristeas

Targum Ps.
Targum Eccl.
80, 87

165
165

On Mating

On the Virtues

325,340

64, 79

79

340

65

65

5




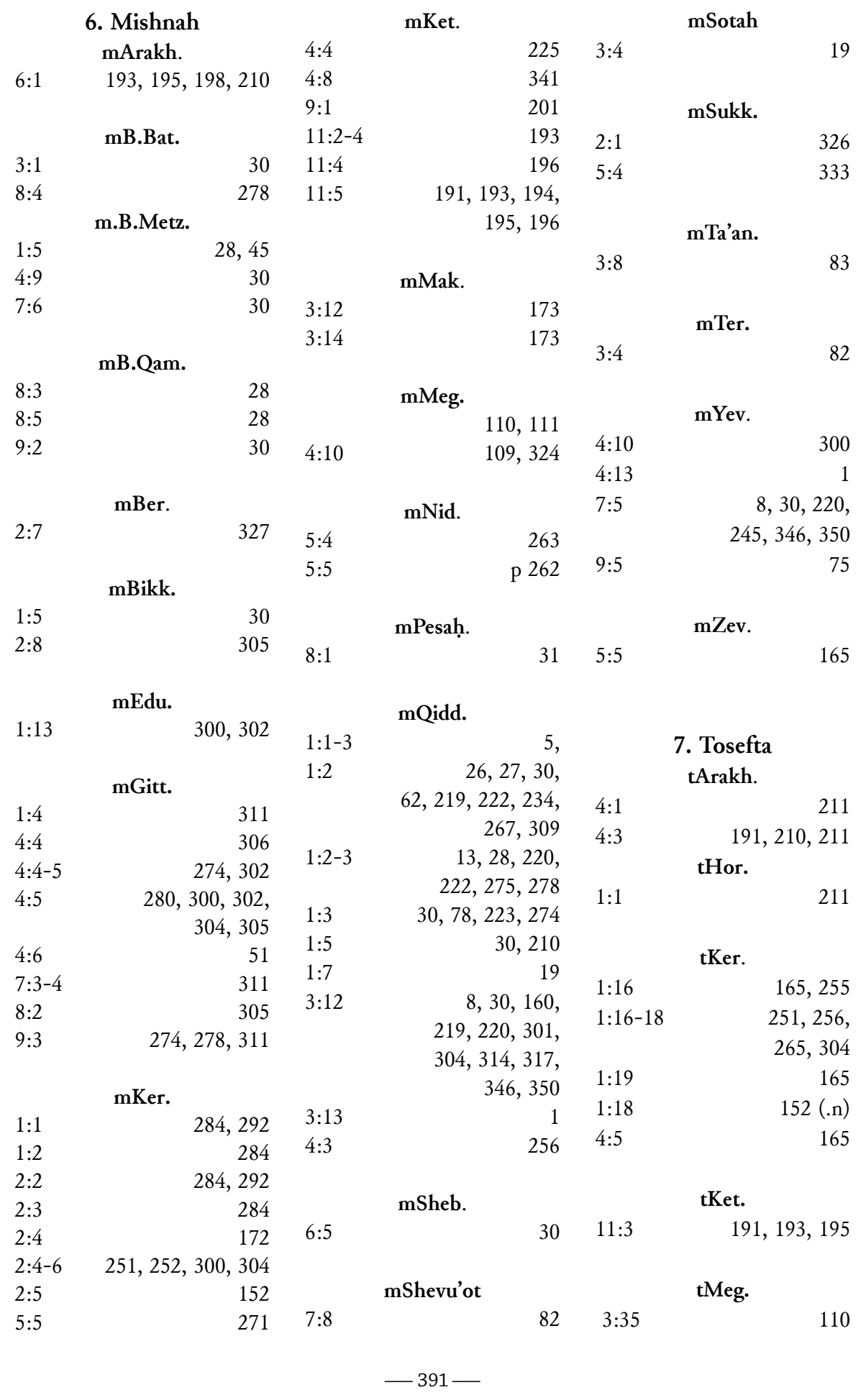


tQidd.

$$
\begin{array}{r}
5: 11 \quad 245,246,248, \\
250,257-260
\end{array}
$$

\section{Midreshei Halakha}

Mekhilta deR. Ishmael (ed. Horovitz)

Neziqin, par. 3

144

Mekhilta deR. Ishmael (ed. Lauterbach)

Dibahodesh (Ex. 20:10) 61

Neziqin par. 226 227, 304

Par. 3 (Ex. 21:7-11) 225, 226, 227, 228

Pisḥa (Ex.23:12) 61, 62

Mekhilta deRashbi

ed. Epstein\&Melamed

Mishpatim

$21: 7$

228

Sifra (ed. Weiss)

Metzora

pereq $6: 1$

262

$6: 7$

262, 263

$7: 1$

262,264

Aharei Mot

$\begin{array}{ll}7: 9 & 270 \\ 9: 8 & 104 \\ 12: 1 & 270 \\ 13: 18 & 269\end{array}$

Emor

par. 5:4-5

(a)

par. 6:2-3

BeHar

Qedoshim

pereq 5

$$
\begin{aligned}
& 172,260-262, \\
& 267,269,272,
\end{aligned}
$$

274, 275, 279-284, 286-291, 294, 295, 298-302, 304-306,

312

$5: 2-4$

$5: 10$

$9: 13$

$9: 14$

$10: 13$

\section{Hova}

pereq $21: 7$

Sifrei Deut.

pisqa $118 \quad 229,231,236$

Sifrei Num. (ed. Horovitz)

pisqa 78

pisqa 117

86

85

Sifrei Zuta (ed. Horovitz

Num. 12:5

89

Midrash Tannaim

230, 236, 237

9. Minor Tractates

$$
\begin{array}{cr} 
& \text { Semahot } \\
1: 13-14 & 326,332,344
\end{array}
$$

65

$7: 838 a$

yShevu'ot

ySan.

$1: 219 \mathrm{~b}$

$10: 228 \mathrm{~d}$

yQidd.

152, 165, 168

144, 145

43

30

246, 247

199

86

yYev.

$6: 17 \mathrm{~b}$

$7: 48 \mathrm{~b}$

$9: 510 \mathrm{~b}$

165

75

75

11. Babylonian Talmud bBekh.

$34 a$

145, 147

bBer.

$16 b$

327,332

bGitt.

$6 \mathrm{~b}$

118

$38 \mathrm{ab}$

215,216

$39 \mathrm{~b}$

280, 281, 282

10. Jerusalem Talmud

yBer.

$41 \mathrm{~b}$

273, 276

42b-43a

76

$9: 113 a$

85

$43 \mathrm{a}$

165

bHor.

yGitt.

$$
\text { 215, } 216 \quad 13 b
$$

298

bKer.

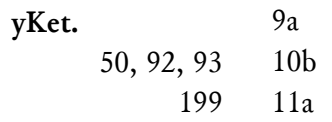

$11: 634 \mathrm{c}$

yNaz.

$165 \quad 11 \mathrm{ab}$
152, 165, 169, 251, 279, 293, 299, 303 288 $8: 157 a$ 
$12 \mathrm{~b}$

$25 \mathrm{~b}$

bKet.

$99 \mathrm{~b}$

$100 \mathrm{~b}$

$\begin{array}{lrr} & \text { bMeg. } & \\ 25 \mathrm{ab} & & 100 \\ & & \\ & \text { bSan. } & \\ 21 \mathrm{a} & & 92,93 \\ 31 \mathrm{~b} & & 86 \\ 103 \mathrm{~b} & & 114\end{array}$

$55 \mathrm{~b}$

$72 \mathrm{a}$

$135 b$

$48 b$

$3 b$

$21 \mathrm{~b}$

$23 a$

$48 \mathrm{~b}$

$55 \mathrm{ab}$

$78 \mathrm{~b}$

$99 \mathrm{a}$

$6 a$

$14 \mathrm{~b}$

$16 \mathrm{a}$

$16 \mathrm{~b}$

$17 \mathrm{~b}$

$18 \mathrm{ab}$

$18 \mathrm{a}$

$18 \mathrm{~b}$

$19 \mathrm{a}$

$20 \mathrm{a}$

$69 \mathrm{a}$

165

165

194, 198

198

86

bShab.

110

165

76,77

bShevu'ot

bSotah

242

19

bYev.

9

62

165

241-243

245-247, 249

bQidd.

160, 165, 300

27

234, 274

241, 243, 244

268

141, 148

145, 230, 231,

233-236

146, 147, 148

147

234

325
$48 \mathrm{a}$

$54 \mathrm{~b}$

12. Midreshei Aggadah

Ber. Rab.

(Theodor-Albeck)

52:1-2

$66: 4$

$84: 2$

bZev.

Gen. Rabbah (Rom) 44:11

$45: 2$

$47: 5$

$61: 4$

$62: 3$

$66: 4$

$70: 7$

$74: 14$

$84: 11$

Bereshit Rabbati

(29:24)

Lev. Rabbah (Rom)

$1: 13$

$12: 1$

$12: 1$

Eccl. Rabbah

7:3-4

Esther Rabbah (Rom)

$5: 1$
Exod. Rabbah (Rom)

$165 \quad 15: 18 \quad 85$

$165 \quad 35: 6 \quad 85$

$40: 4 \quad 318$

43:6 83

Midrash Leqaḥ Tov

(Rom)

Lev. 19:20

173

Midrash Sekhel Tov

Gen. 35:22 97

Midrash Tehilim

79

170

$24: 2$

85

$4: 3 \quad 85$

55

90

54

326

331

Midrash Tehilim (Vilna 1891)

2:13

85

Num. Rabbah

$4: 1 \quad 85$

$34210: 1 \quad 157$

$343 \quad 14: 11$

328,333

340

21:15

85

$$
\begin{array}{r}
334,336,337, \\
338,339,341, \\
342,343
\end{array}
$$

91

84

(parshat 27,3)

64

Pirqei de R. Eliezer pereq 37

344

84

329

Sefer ha-Yashar

341

77

$1: 12 \quad 329,330$

Song of Songs Zuta

84

$1: 15$

328 
Vayeshev $2 \quad 114$

Ki Tissa siman $13 \quad 318$

Yalqut Shimoni

remez 320/Ex. 21

Mishpatim Ex. 22

Re'eh remez 88/Deut. 15

232

$$
\begin{array}{cr}
\text { Midrash HaGadol } \\
\text { Exod 12:36 } & 241,244, \\
& 260,261, \\
262,290
\end{array}
$$

\section{Commentators}

Abarbanel

93

Ibn Ezra 160, 169, 239, 267

Malbim

$$
273,280 \text {, }
$$$$
\text { 283, } 285
$$

Maimonides (Guide)

Magid Mishneh

16

Metzudat David

59

Moshe haDarshan 314, 328, 332,334 , 342,343

Nahalat Ya'aqov 332

Nahmanides

$$
\begin{array}{r}
55-57,93, \\
94,169,174,
\end{array}
$$

Penei Moshe

82,216

Rabad

94, 95, 260, 261, 263, 279

Rabbenu Bahya 333

Rabbenu Hillel

$$
\text { 260, 261, }
$$$$
269,279
$$

Radak

60, 102

Rashak

255

Rashba 146, 147, 231, 235

Rashbam

Rashi

$$
\begin{array}{r}
239 \\
59,67,86, \\
92,93,101, \\
103,116,126, \\
145-148,161,
\end{array}
$$

173, 198, 242, 244, 267, 285,

B3.11

80,323 $325,327,342$,

343

Qorban Aharon

289

Qorban HaEdah

75,216

Tosafot

20, 301

Tosafot HaRosh

148

Yaqub al-Qirqisani

249

14. Posqim

Mishnah Torah

Avadim

$7: 1-2$

$7: 6$

$8: 13$

$9: 3$

$4: 16$

$3: 13$

$3: 14$

$3: 17$

12:11

12:13

$12: 25$

$15: 4$

Malveh veLoveh

12:11

197

Talmud Torah

1:13

Yam shel Shelomo

Qiddushin

278

281

251

244

Ishut

161

Issure Biyah

161

173

263

325

325

240

9, 325

$914: 6$

91:20

$89-4-26,6$

BU

Aug. 65

BE

81

80

167

$\mathrm{CH}$

50

CT

$626 a$

II. 9-12

19

II 39

VIII 22b

VIII $28 \mathrm{~b}$

3334

4740

4853

5321 and Texts

Aramaic Documents

(Porten/Yardeni)

2.2, 11

2:B.3.3

202

320

B. 2.2

321

B.3.5

321

$310,320,321$

B. 3.6

Cyr.

Dar. 


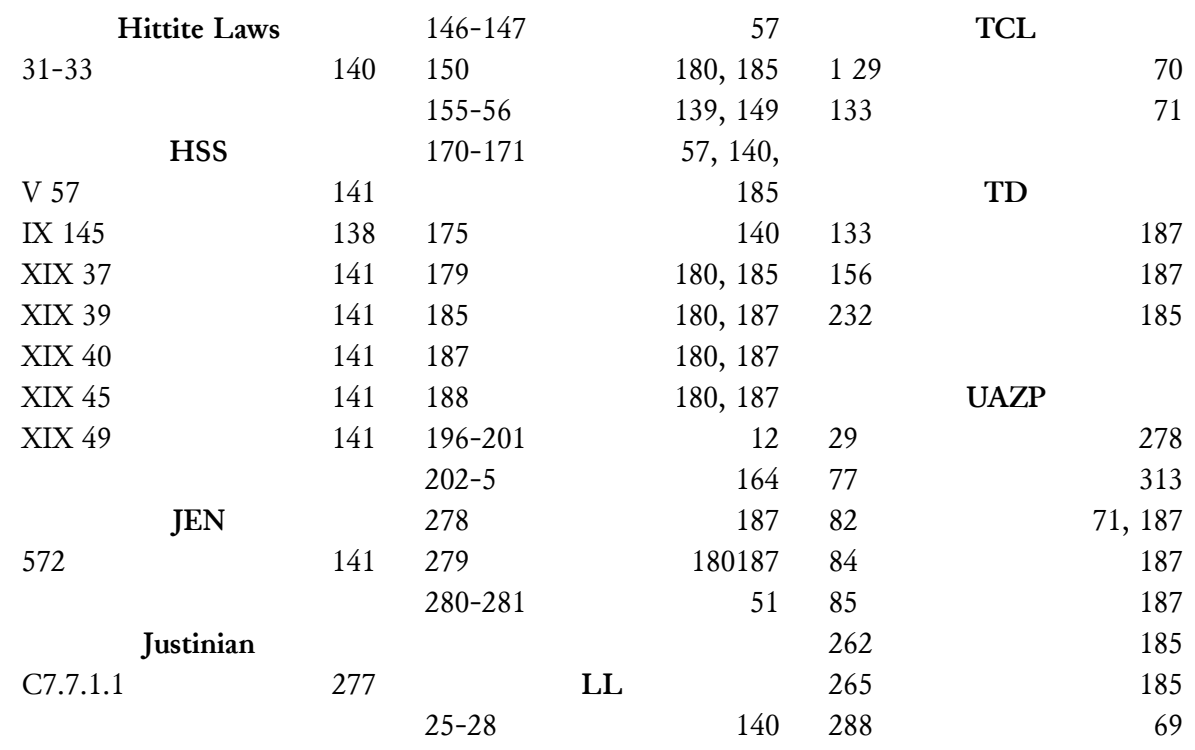

492
630
958
1097

1097

K

$\begin{array}{rr}167 & \\ 168 & 5 \\ 72 & \\ 73 & \end{array}$

LU

$140 \quad 6143$

VAB

MAL

Keret Epic

K III iv 13

$\mathrm{Ki}$

1902-5

1902-10

1902-13

LE

$\begin{array}{rrr}31 & 154,158,217 \\ 42 & 164 & 6: 102\end{array}$

LH

9-13

114-116

115-119

116

117

117-119

118

125

129

A 43

A 44

$\mathrm{Ki}$

$1,80,18$

73

73

73

N. 432

NII, 120

Nuzi

MSL

(1)

PSBA

$13916,4: 25$

66

V 73

$179 \quad \mathrm{~V} 85$

V 126

VII 50

140

140

16. General Index

actio in personam 182

187

actio in rem

155, 178, 182-184, 190

SAA

73,74

adulterer/adulteress 14, 155,

$\begin{array}{rlr}11,184 & \text { I, 99 } & \\ 66 & 5,199 & \\ 162 & \text { X 316 } & \\ 67 & 10,191 & \\ 136,183 & \text { XI } & \\ 66 & & \\ 180 & & \text { SAAS } \\ 11,184 & \text { V 16, BM 103206 } \\ 13,264 & \text { V }\end{array}$

168

73

167

72,73

308

72
70

1

87

87

85

78

13

187

87

85

69

70

69

187

187

187

187 
ah

13, 21, 125, 127, 221, 222,

224, 347

ahim

28

amah

$4,5,12$,

$16,21,26$,

$29,33,36-46$, 48-62, 66, 68, 80, 87, 90, 94,

95, 96, 98, 109, 116, 121-125,

127-128, 129, 130-136,

142, 148-150, 152, 219, 220, 222-225, 226, 227-231, 237, 249, 250, 267, 269, 310, 315, $317,318,320$, 328, 343, 344, 345, 346, 347 amtum $46,57,69$, 70,154 avadim

$37,61,122$, 126, 134, 226, 227, 228, 229, 336

awilu

ba'al marriage

beena

begged

begidah

ben amah

ben bayit

ben horin Bilhah

\section{2}

45,94

$45,46,58$, $94,116,117$ 145, 146 145

$33,53,54$, 55-60, 62, 87, 90, 94, 249

$33,53,65$, 78-80, 82-87, 238 4, 26, 31 34, 36, 40, $41,43,56$,
$89,90,95$,

319,325

98, 99, 100, 101, 104-111, 118, 249, 313-319, 324, 326, 328-330, 332-344

biqqoret

14, 33, 151-153,

155, 158, 160, 161, 162,

171-175, 177, 178, 181-184, 191-200, 202, 203, 205, 208-212, 216, 217, 279, 285

$\mathrm{BQR} / \mathrm{PQR} \quad 33,138,152$, 157, 174, 175, 178, 179, 181, 183-185, 187, 188, 190-192, 213, 216, 217 Decalogue 36, 60-62, 133 Eshnuna 23 eved $4,5,12,16$, 21, 26, 28, 30, 31, 36-38, 42, 44, 50, 52-54, 57, 59-62, 64, 66-68, 80, 82, $90,122,123-127$, 128, 130-136, 140, 223, 224, 226, 227, 229, 230, 237, 269, 299, 304, 309, 346, 347 eved ivri/Hebrew slave 122, 123, 132, 223, 224, 226, 229, 230 , 233-235, 297, 347

Canaanite slave $28,38,161$, 223, 226, 239, 244, 256, 266, 267, 274, 275

cessio in iure 277 5, 6, 7, 17, 20-23, 32,

155, 348 comparative law 16,347 concubine $\quad 4,33,35,36$, $39,40,41,50$, 52, 53, 89, 90, 91-96, 98, 99, 101, 102, 103, 104, 110, 113, 114, 115, 121, 150, 170, 300, $315,318-320$, 323, 343-345 28, 229, 259, 304 eved olam 21, 122, 124, 128, 132, 133, 135, 136, 140, 221, 226, 309

free man

299

freedman 247

functional equivalence/

equivalents

15-17, 26, 33, 62, 132, 137, 139, 150, 155, 347

gentile/s 8, 9, 78, 127, 245-48, 250, 270, 301, 350

concubinage

5, 38, 39, $89,91,92$, 95, 96, 103, 104, 121, 162, ger

ger tzedeq ger toshav Hagar
61, 214, 247

61

61

36, 40, 41, 54, 


$\begin{array}{rrr}56,90,98,99, & \text { lectio difficilior } & 142,147, \\ 105,170, & 232,235, & \\ 313-315 & 236,249, & \\ 326 & 288,332 & \end{array}$

Haggada

half slave-half free 268,276 , 279, 303,

305

Hammurapi

$10,12,17$ 23, 50, 56,

57

Hand wahre Hand 11184 hapax/es

$13,14,33$, 41, 152, 155, 159, 163, 172,

177, 217

heqdesh

191, 210, 211

heres

277292

hevqer/hefqer

192, 212,

213, 217

ḩofshi

4, 26, 31

Holiness Code 128, 156

iggeret biqqoret $\quad 175$

191-200,

202, 203,

208-210,

211, 212

iggeret mazon 192, 199, 206

inter vivos

277

ishah

4, 36, 37,

54, 90, 91,

94, 97-99,

$105,111,112$,

116, 119, 162,

315, 317, 345, 348

ishah pilegesh

90, 111,

112,116

Josephus

$44,65,90$,

114, 228, 249,

$317,324,325$,

344

Jubilees $\quad 64,79,340,344$

Juridicization

15

LB

$81,167,179$,

187, 188, 308

LE

155 lex talionis

$\mathrm{LH}$

mamzer/mazerut $8,9,220$, 245, 247-250,

350,351

mār ālim

mār bīti

mār bītim

mār ekallim

māru/ mārū

matrilineal

7, 9, 34, 53,

121, 150, 158,

219, 220, 237,

245,250

matrilineal principle

6-10, 30, 33,

34, 52, 127,

152, 158, 218-220,

314, 346, 348,

349, 350352

matrilocal

45,46

meshuhrar/reret

4, 26, 30

31, 247, 349

MB

178, 179

NA

NB

51, 57, 72,

73, 167, 168,

201, 308, 313

$66,72,81$,

137, 179, 201,

308

Neo-Sumerian

neherefet

308

10, 13, 33, 41,

151-153, 155,

159-161, 162,

166-170, 183,

217, 251, 252,

265, 267, 280,

284, 288, 290,

297, 299, 300,

301, 304, 350,

netinim
$\mathrm{OA}$

$\mathrm{OB}$$$
\mathrm{OB}
$$

24, 69, 71

$72,78,81$,

138, 140, 152,

178, 179, 184,

185-187, 278, 313

Philo

249, 250261 ,

325,340

pilegesh

4, 33, 35,

$50,57,58$,

89-109, 111,

112, 113,

114-119, 170,

$315,318,323$,

324,344

PLH 141, 309, 310

qiddushin

$8,92,93$,

142, 170, 225,

245, 301, 304,

314, 348

qinyan

5,95

qinyan kesef $\quad 75,76,78$

Qumran

5, 34, 105, 107, 334, 335

sakhir

Sarah

238

54, 56, 101, 313, 326-330,

$332,333,340$

sex right

4-6, 10,

32, 34, 251, $345,348,350$,

352

shifhah

1, 4, 5, 10, 16, 26, 27, 28, 30, 33, 36-41, 42, 43-46, 47, 48, 52, 54, 56, 77, 82, 89-91, 94, 95, 96, 109, 142, 151, 152, 153, 157, 158, 161, 162 163, 


\begin{tabular}{|c|c|c|c|c|}
\hline $\begin{array}{l}169,170,172,217, \\
218,224,227,251,\end{array}$ & $\begin{array}{l}\text { shinnui ha- } \\
\text { talion }\end{array}$ & $\begin{array}{r}352 \\
11,12,13\end{array}$ & $\begin{array}{l}237-244 \\
\text { vindictam in }\end{array}$ & ponere \\
\hline $252,254-256,258$ & & $68,123,226,350$ & virilocal & $45,46,116$ \\
\hline $259,263,265$ & Targum & $34,39,43$ & wilid bītim & $63,68-72,78$ \\
\hline $267-270,280$ & & $54,56,60$ & wildum & 69 \\
\hline $282,284,288-294$, & & $62,79,80$ & yelid bayit & $33,53,57$, \\
\hline $297,299,301-304$, & & $87,89,100$ & & $59,60,62-66$ \\
\hline $314,315,317,318$ & & 102,106 & & $68,69,71$ \\
\hline $337,338,342$ & & $109-111,116$ & & $74-78,87$ \\
\hline $344-346,350$ & & $150,160-162$ & Yizkor & 326 \\
\hline shifhah kena'anit 1,28 , & & $165,172,176$ & Zilpah & $34,36,40$ \\
\hline ah neherefet $10,13,41$, & & $192,193,238$ & & $41,43,56$ \\
\hline 142 151, 161, & & $269,275,309$ & & $98,105,109$ \\
\hline $162,169,170$ & & $314,317,318$ & & $249,313-319$, \\
\hline $251,252,265$, & & 320323,324 & & $324,326,328$, \\
\hline $267,280,284$, & & $325,331,333$, & & $329,330,332$, \\
\hline $288,290,297$, & & 343,344 & & $333,335-344$ \\
\hline $301, \quad 304$, & terumah & $8,75,76,82$ & zonah & 116,117 \\
\hline 350 & toshav & 61 126, 127, 221, & & \\
\hline
\end{tabular}



